\title{
Experimental study of the stress state and strain rate dependent mechanical behaviour of TRIP- assisted steels
}

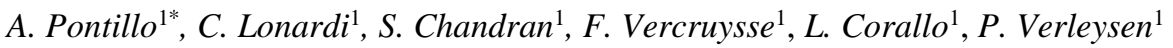 \\ ${ }^{1}$ Department of Electromechanical, Systems and Metal Engineering, MST-DyMaLab Research \\ Group, Ghent University, Technologiepark 46, 9052 Zwijnaarde, Belgium
}

\begin{abstract}
This paper presents an investigation into the effect of different stress states and strain rates on the austenite-to-martensite transformation during plastic straining of a Q\&P steel. Different stress states are imposed to the steel using purposed-designed samples. The sample geometries, including in-plane shear, dogbone and plane strain samples, are optimised by finite element modelling. Tensile tests are performed at different strain rates of $0.001 \mathrm{~s}^{-1}, 10 \mathrm{~s}^{-1}$ and $500 \mathrm{~s}^{-1}$. Digital image correlation is used to capture the strain fields during the entire deformation process. The mechanical results indicate a positive strain rate sensitivity for both the shear and plane strain specimens and a negative strain rate sensitivity for the dogbone sample. In addition, the influence of the strain rate on the strain level is more pronounced for the shear than for the plane strain specimens and for the dogbone samples.
\end{abstract}

\section{Introduction}

The trends in advanced high strength steel (AHSS) development for the automotive industry are driven by ever more stringent rules for both safety, fuel consumption and $\mathrm{CO}_{2}$-emissions. To meet the regulations, carmakers are constantly striving to reduce car weight and, at the same time, to reach higher levels of crashworthiness [1]. In the last few years, a third generation of AHSS is being developed, exhibiting better performance than the previous generations. The third generation includes quenched and partitioned (Q\&P) steels that rely on the transformation induced plasticity (TRIP) effect, i.e., the transformation from (retained) austenite (RA) to martensite during plastic deformation. The TRIP effect enhances properties such as strength, ductility and energy absorption. Therefore, these properties make these steels especially suitable for automotive applications [2].

Recent TRIP-assisted steel developments target an optimal mechanical stability of the RA. The mechanical stability of RA is not only determined by material intrinsic parameters, such as chemical composition, grain size and morphology, though also by loading conditions such as stress state, strain rate and deformation temperature [3],[4]. Experiments on carburized 4320 steel indicate that the most suitable stress state for stress-induced transformation of austenite is tension rather than compression [5]. In fact, compressive loading suppresses the

* Corresponding author: Antonio.Pontillo@ugent.be 
transformation and stabilizes the retained austenite as pressure hinders the volume expansion which accompanies the transformation. A series of experiments on $18 \mathrm{Cr}-8 \mathrm{Ni}$ austenitic stainless steels shows that deformation by tension causes more formation of martensite than torsion and compression [6]. Several researchers have shown that an increase in stress triaxiality leads to an increase in martensite transformation rate [7],[8]. Regarding the effect of the strain rate on the mechanical stability, it is known from literature [9] that, for Q\&P steel, the stability increases with the strain rate at lower strain rate levels $\left(<0.1 \mathrm{~s}^{-1}\right)$, while at higher strain rates $\left(>1 \mathrm{~s}^{-1}\right)$, the stability decreases with the strain rate. Moreover, at dynamic strain rates, the deformation occurs under adiabatic conditions. All heat generated in the material results in a temperature rise which increases the austenite stability and suppresses the TRIP effect [10].

Present work aims to investigate the effect of strain rate and stress state, more specifically stress triaxiality, on the martensite formation and mechanical properties of third generation TRIP-assisted steels. For this purpose, static, intermediate and dynamic tensile tests are performed on a Q\&P steel using three different sample geometries. Aiming at a wide range of stress triaxialities, a shear, a uniaxial tension and a plane strain specimen are considered. The specimen geometries are optimised using finite element simulations aiming at an as constant as possible stress triaxiality in a specific region of the sample. The sample geometries are tested at static, intermediate and dynamic strain rates using a conventional static test bench and a split Hopkinson bar tensile setup. In order to capture the highly heterogeneous strain fields during testing, static and high-speed camera recordings of a speckle pattern applied on the sample surfaces are processed by Digital Image Correlation (DIC).

\section{Material and methods}

\subsection{Material}

The studied Q\&P steel has a nominal composition of $0.2 \mathrm{C}-1.25 \mathrm{Si}-2.4 \mathrm{Mn}-0.02 \mathrm{Al}-0.025 \mathrm{Cr}$ (wt $\%$ ). The base metal was cast, hot-rolled and cold-rolled into a sheet with a final thickness of $1.5 \mathrm{~mm}$. The imposed Q\&P heat treatment cycle aimed at maximising the fraction of retained austenite and minimising the formation of fresh martensite during the final cooling to room temperature. After the Q\&P cycle the amount of retained austenite, derived using electron beam scatter diffraction (EBSD), is 5\% [4].

\subsection{Experimental methods}

\subsubsection{Specimen geometry optimisation}

Three different specimens are used for the current investigation: a uniaxial specimen, a plane strain specimen and in-plane shear specimen. The uniaxial sample is a traditional dogbone geometry with dimensions suitable for Split Hopkinson Tensile Bar (SHTB) testing. Geometrical specification of dogbone specimen are presented in [4]. A schematic presentation of the proposed plane strain and shear sample is given in Fig. 1. Both geometries are sufficiently small to be tested using split Hopkinson bar tensile setups in order to ensure the achievement of force equilibrium in dynamic testing. Indeed, the length between the pins, used to transfer the tensile load, is smaller than $40 \mathrm{~mm}$. 

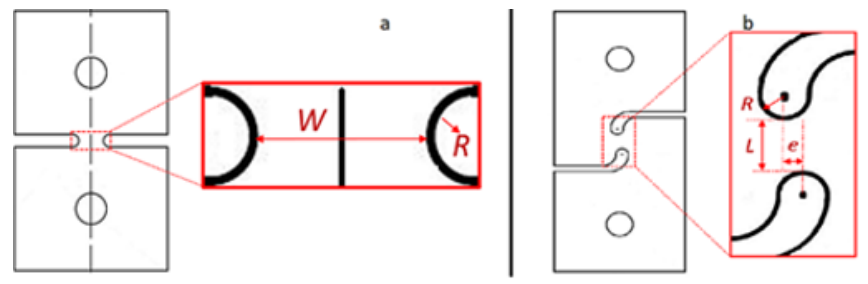

Fig. 1. a) Plane strain specimen and b) in-plane shear specimen

A finite element-based optimisation cycle is implemented in order to find the critical dimensions (red variables in the red boxes in Fig. 1) that yield the desired stress states. Here, the aim is to have an as high as possible stress triaxiality for the plane strain specimen and a low $(\approx 0)$ stress triaxiality for the in-plane shear specimen. Moreover, the sample geometry is optimised to have the desired, constant stress triaxiality in a sufficiently large region, i.e., a cube with $1 \mathrm{~mm}$ side, whose centre coincides with the centre of the sample during the plastic deformation, see Fig. 2 . The analysis focuses on the low deformation region from onset of deformation till a mean plastic equivalent strain of $10 \%$ is reached in the cube. The sample geometry optimization is performed using a Python script based on the synergy between MATLAB and Abaqus using the Levenberg-Marquardt algorithm. The optimised dimensions for each specimen are shown in Fig. 3.

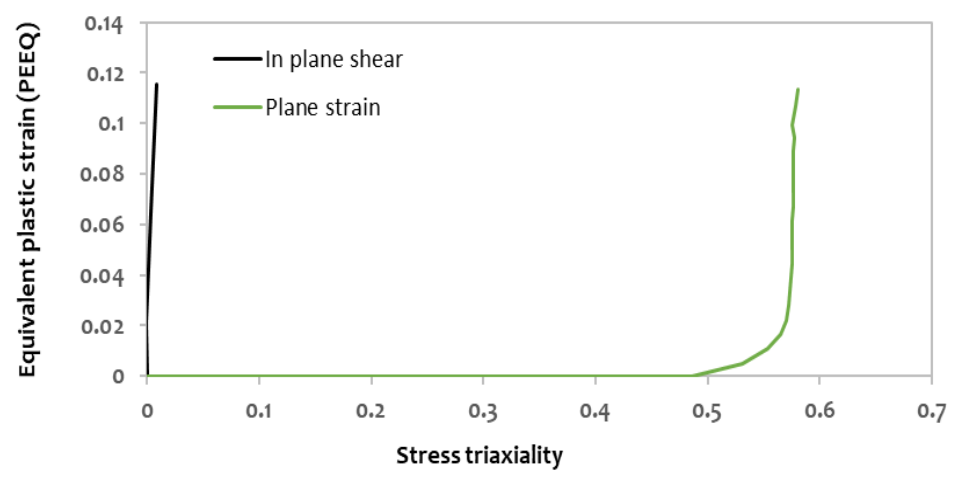

Fig. 2.: Evolution of the stress triaxiality in the sample centre as a function of the plastic equivalent strain for the plane strain and in-plane shear geometries
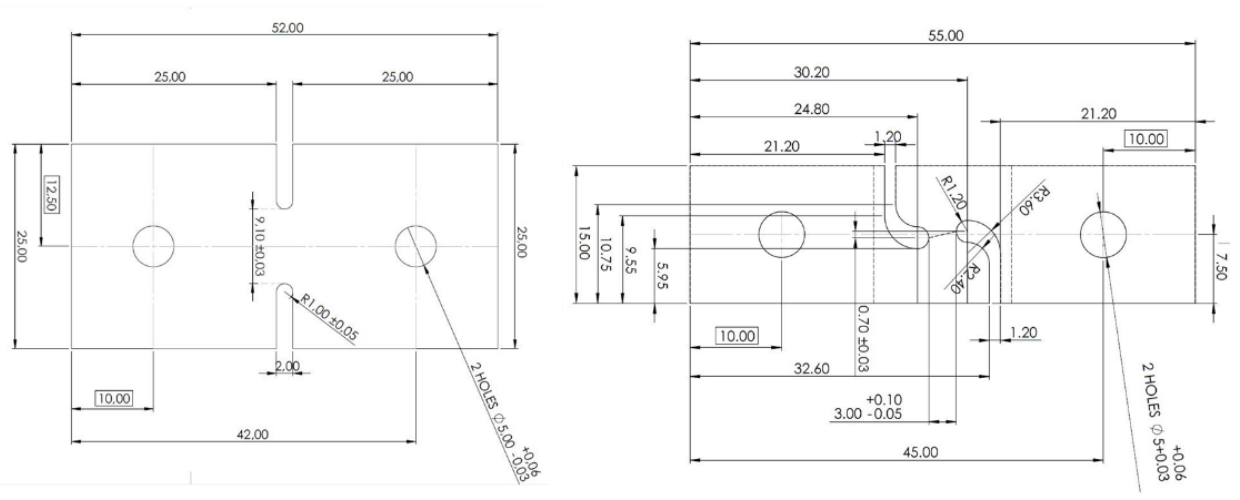

Fig. 3. a) Plane strain specimen geometry b) In-plane shear specimen geometry 


\subsubsection{Tensile test setups and measurements techniques}

To characterise the quasi-static (strain rate of $0.001 \mathrm{~s}^{-1}$ ) mechanical properties, an Instron 5569 tensile test device is used. For the dynamic properties, aiming at a strain rate of approximately $500 \mathrm{~s}^{-1}$, the SHTB setup of Ghent University is used [4],[11]. For the plane strain specimen, intermediate strain rate tests at $10 \mathrm{~s}^{-1}$ are performed using the Instron device. For each specimen and deformation rate, three tests are performed. Identical sample geometries and boundary conditions are used in the static, intermediate and dynamic tests. Therefore, differences between the results for a specific sample geometry can be attributed solely to differences in strain rate. The tests are performed at room temperature. To obtain reliable strain values in the region of interest, static and high-speed imaging combined with the DIC technique is used. To this purpose, prior to testing, a black and white speckle pattern is applied to the sample. Two cameras, 5Mpxl Stingray and Photron Fastcam Mini AX2000, for the static and dynamic experiments respectively, are used to capture the deformation of the speckle pattern during the tensile experiments. To calculate the evolution of the strain fields during the tests, the recorded images are processed by the commercial DIC software MatchID. The strains reported in section 3 are calculated in the centre of the gauge section.

\section{Results and Discussion}

\subsection{Mechanical test results}

Representative stress-strain curves of all tests are shown in Fig. 4. Both shear and plane strain specimens show an increase in the stress level with an increasing strain rate, see Fig. $4 a$ and Fig. $4 b$. Indeed, a positive strain rate sensitivity from static to dynamic tests and from static to intermediate tests is observed for the shear and plane strain tests, respectively. Conversely, the uniaxial dogbone specimen shows an opposite trend, see Fig. $4 c$. Indeed, at high strain rates, especially at large deformation levels, a significant decrease in stress levels is observed. In line with expectations, the experiments confirm that an increase in stress level is obtained from shear over the dogbone to the in plane strain specimen due to the increase of stress triaxiality.

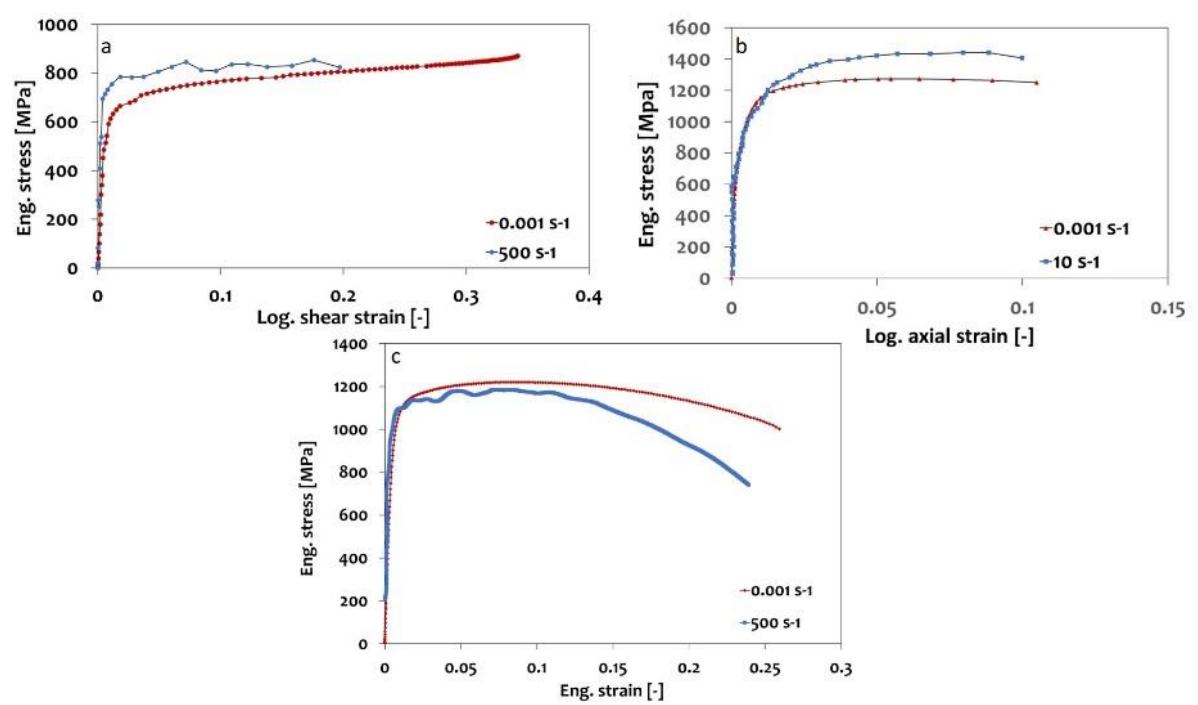

Fig. 4. Representative stress-strain curves of a) static and dynamic in-plane shear tests, b) static and intermediate plane strain tests and c) static and dynamic tests on dogbone samples 
Contour plots of logarithmic axial strains and logarithmic shear strains just before fracture, together with the initial frame, are presented in Fig. 5 for a plane strain and shear test. Regarding the influence of the strain rate on the strain levels, the local logarithmic shear strain is around $20 \%$ for dynamic tests and around $35 \%$ for the static. Similar results are reported in [11]. The difference between static and intermediate logarithmic axial strain decreases for the tests on the plane strain specimens, see Fig. $\mathbf{4 b}$. Indeed, for the static tests, the logarithmic local strain is around $10.5 \%$, whereas for intermediate tests, it is around $10 \%$. In dogbone samples, the engineering strain is around $26 \%$ and $24 \%$ for the static and dynamic test respectively. Furthermore, the difference in the engineering strain is less pronounced in the tensile specimen in comparison with shear samples.

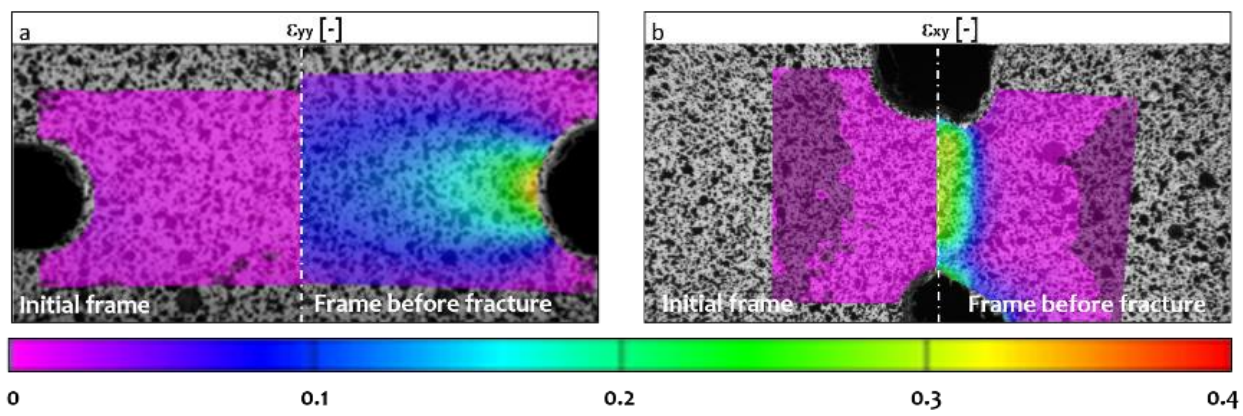

Fig. 5. Local strain measurement for a) a plane strain test and b) in-plane shear test, both performed at $10^{-3} \mathrm{~s}^{-1}$

\subsection{Discussion}

Uniaxial tests are generally used to identify the TRIP effect in AHSS [3],[9],[10]. For the Q\&P steel, starting from an initial EBSD based-fraction of $5 \mathrm{wt} \%$ of retained austenite, 0.65 $\mathrm{wt} \%$ is left untransformed, in dogbone samples, under dynamic loading as opposed to 0.5 $\mathrm{wt} \%$ under static strain rate [4]. As a result, a negative strain rate sensitivity is observed under uniaxial stress states. The suppression of the TRIP effect under dynamic loading conditions is attributed to adiabatic heating. In comparison, for the plane strain specimen, the increase of stress triaxiality, which could potentially better accommodate the volume change needed for the austenite transformation into martensite, causes the adiabatic heating to have a less pronounced effect on the RA stability. Similarly, for the shear stress state, an even more positive strain rate sensitivity is found which might indicate that, next to stress triaxiality, also the Lode angle is expected to influence the martensite transformation. Thus, a complex transformation kinetics is perceived that is highly dependent on the stress state. Attempts were made to link the mechanical test results with the austenite content in the centre of the sample after fracture. However, this proved to be difficult, yet impossible, for the shear and plane strain samples. Indeed, while for the uniaxial samples a considerable part of the gauge section, i.e., next to the neck region, is uniformly deformed and can thus be used to quantify the austeniste content after deformation, this is not the case for the two other samples where high strain gradients occur around the centre of the sample. Consequently, X-ray diffraction (XRD) or EBSD measurements do not provide conclusive results. Therefore, in the future, tests will be carried out that are interrupted at well-chosen, low levels of deformation in order to allow to quantify the transformation kinetics. Further studies will focus on the correlation between the amount of retained austenite, deformation level, stress state and strain rate. 


\section{Conclusions}

To elucidate the influence of the stress state and strain rate on the mechanical behaviour of TRIP-assisted steels, an experimental campaign was designed including static, intermediate and dynamic tests using three different sample geometries. The sample geometries were optimised aiming at specific stress states in a $1 \mathrm{~mm}^{3}$ cube around the centre upto equivalent strains of $10 \%$. The experimental design was successfully applied for a Q\&P steel. Following conclusions were obtained from the research:

- A suppression of RA transformation is observed with an increasing strain rate for the dogbone sample which is attributed to adiabatic sample heating at dynamic strain rates.

- Test results suggest a potential rise in martensite formation, or an enhanced TRIP effect, under plane strain conditions. Compared to the dogbone specimen, the high stress triaxiality might more easily accommodate the volume change needed for austenite to transform during straining.

- The shear tests show a possible influence of the Lode angle. The combined influences of stress triaxiality and Lode angle might result in complex austenite transformation phenomena.

- The test campaign clearly shows the need for interrupted testing in order to obtain reliable data on the transformation kinetics.

The authors gratefully acknowledge the financial support received from the European Union's RFCS programme via the project OptiQPap, No. 899482.

\section{References}

[1] N. Fonstein, Advanced High Strength Sheet Steels. Cham: Springer International Publishing, (2015).

[2] R. Colás and G. E. Totten, Eds., Encyclopedia of Iron, Steel, and Their Alloys. CRC Press, (2016).

[3] D. De Knijf, C. Föjer, L. A. I. Kestens, and R. Petrov, Materials Science and Engineering: A, vol. 638, pp. 219-227, (2015).

[4] F. Vercruysse, C. Celada-Casero, B. M. Linke, P. Verleysen, and R. H. Petrov, Metals, vol. 10, no. 4, p. 509, (2020).

[5] I. Karaman, M. Balzer, H. Sehitoglu, and H. J. Maier, Metall and Mat Trans A, vol. 29, no. 2, pp. 427-437, (1998).

[6] A. A. Lebedev and V. V. Kosarchuk, International Journal of Plasticity, p. 19, 2000.

[7] P. Jacques, Q. Furnemont, T. Pardoen, and F. Delannay, Acta Materialia, vol. 49, no. 1, pp. 139-152, (2001).

[8] E. Polatidis, W.-N. Hsu, M. Šmíd, T. Panzner, S. Chakrabarty, and P. Pant, Scripta Materialia, vol. 147, pp. 27-32, (2018).

[9] D. Q. Zou, S. H. Li, and J. He, Materials Science, p. 10, (2017).

[10] Z. Gronostajski, A. Niechajowicz, R. Kuziak, J. Krawczyk, and S. Polak, Journal of Materials Processing Technology, p. 14, (2017).

[11] J. Peirs, P. Verleysen, and J. Degrieck, Exp Mech, vol. 52, no. 7, pp. 729-741, (2012). 